Comparison of filter cl eani ng perfor mance bet ween VDI and JIS testing rigs for cl eanable fabric filter

\begin{tabular}{|l|l|}
\hline 著者 & $\begin{array}{l}\text { Nao Ni ng, Yao Yupi ng, Hat a M t suhi ko, Wada } \\
\text { Nasashi, Kanaoka Chi kao }\end{array}$ \\
\hline $\begin{array}{l}\text { j our nal or } \\
\text { publ i cat i on ti t l e }\end{array}$ & Powder Technol ogy \\
\hline vol une & 180 \\
\hline number & $1-2$ \\
\hline page r ange & $109-114$ \\
\hline year & $2008-01-14$ \\
\hline URL & ht t p: //hdl . handl e. net /2297/7673 \\
\hline
\end{tabular}




\title{
COMPARISON OF FILTER CLEANING PERFORMANCE BETWEEN VDI AND JIS TESTING RIGS FOR CLEANABLE FABRIC FILTER
}

\author{
Ning Mao ${ }^{1}$, Yuping Yao ${ }^{1}$, Mistuhiko Hata ${ }^{1}$, Masashi Wada ${ }^{2}$ and Chikao Kanaoka ${ }^{2}$ \\ ${ }^{1}$ Graduate School of Natural Science and Technology, Kanazawa University \\ ${ }^{1}$ Kakuma-machi, Kanazawa, Ishikawa, 920-1192, Japan \\ ${ }^{2}$ Ishikawa National College of Technology \\ ${ }^{2}$ Kitacyujo, Tsubata, Kahoku-gun, Ishikawa, 929-0392, Japan
}

\section{ABSTRACT}

VDI type-1 rig and JIS rig are the two major testing rigs for cleanable fabric filters. We measured the filter cleaning performance using these rigs and the results were compared in order to characterize the two testing methods. The filter performance tests showed that the filter cleaning efficiency measured with VDI type-1 rig is higher than that with JIS rig. During pulse jet cleaning, JIS rig gave a higher peak pressure and a shorter time period of pulse jet compared to VDI type-1 rig. A new filtration model was applied to the filtration cycles with the two rigs and it was shown that the difference in filter cleaning performance measured by VDI type-1 and JIS rigs can be expressed in terms of the surface cleaning fraction, which is the ratio of cleaned surface area to the total surface area of a filter, and the residual dust load on the un-cleaned surface after cleaning.

\section{INTRODUCTION}

Fabric filters are widely used to remove particulate matters in flue gas with a high collection efficiency. In order to evaluate the filtration performance, a number of testing rigs have been developed, in which VDI type-1 rig and JIS rig are the two representative ones. ${ }^{[1-2]}$

Incomplete filter cleaning has a marked effect on the filtration performance of fabric filters where the dust cake is detached unevenly from the filter surface during the periodical filter cleaning. The present paper compares the filter cleaning performance measured by VDI type-1 rig and JIS rig and extracts the essential difference in the testing methods.

There are many models for expressing the filtration process with patchy cleaning ${ }^{[3-5]}$. Some previous models may express the actual dust cleaning pattern on filter surface, but they seem too complicated for practical use. Therefore, a new model is proposed and applied to evaluate and compare the filter cleaning performance measured by VDI type-1 rig and JIS rig.

\section{EXPERIMENTAL SETUPS}

VDI type-1 rig specified in VDI/DIN 3926 and JIS rig in JIS Z 8909-1 are used in the present work. They compose of three parts, i.e. the dust feeding and dispersing part, the dust collecting and cleaning part, and the monitoring and controlling part. The schematic diagrams of VDI type-1 rig and JIS rig are shown, respectively, in Figs. 1 and 2.

Test dust from a two-stage screw dust feeder is dispersed by an ejector. The dust collecting and cleaning part includes a vertical duct and a horizontal duct. The dust laden gas is introduced 
into the top of the vertical duct, and the dust in the gas is collected on the testing filter installed at the inlet of the horizontal duct. The filter pressure is recorded by a pressure transducer (VALCOM, VPRN-A4). The filtration-cleaning cycle controlled by either a prescribed filter pressure or time interval is repeated at a given filtration velocity. When the filter pressure reaches a prescribed value (1000 $\mathrm{Pa}$ in the present work), filter cleaning is carried out with compressed air of $500 \mathrm{kPa}$-gauge through a solenoid valve connected to a $2.5 \mathrm{~L}$ compressed air reservoir. Otherwise, filter cleaning is carried out with a preset time interval ( $5 \mathrm{~s}$ in the present work) for a large number of cycles. The downstream dust concentration is measured by sampling the test particles with HEPA filters.

The JIS rig is small in dimension compared to VDI type-1 rig. Since all the dust laden gas passes through the test filter in JIS rig, it is called as total-flow type. However, in VDI type-1 rig, only a small fraction of gas passes through the test filter and a larger fraction of gas leaves from the rig at the bottom of the vertical duct. Therefore, VDI type-1 rig is called as part-flow type rig. The test filter used in JIS rig is rectangular in shape with the size of $300 \mathrm{~mm}$ (width) $\times 300 \mathrm{~mm}$ (length), whereas the filter is circular with $140 \mathrm{~mm}$ in diameter in VDI type-1 rig.

\section{RESULTS AND DISCUSSIONS}

Filter performance test

The filter performance test is conducted using JIS and VDI type-1 rigs. The testing procedure is as follows:

Step 1: Virgin performance test period: 30 filtration-cleaning cycles with a prescribed cleaning pressure drop $(1000 \mathrm{~Pa})$

Step 2: Aging condition period: 5000 cycles with filter cleaning at given interval of $5 \mathrm{~s}$

Step 3: Stabilizing period: 10 filtration-cleaning cycles with the prescribed cleaning pressure drop $(1000 \mathrm{~Pa})$

Step 4: Performance test period: 30 filtration-cleaning cycles with the prescribed cleaning pressure drop $(1000 \mathrm{~Pa})$

The testing conditions are listed in Table 1.

The pressure drop after filter cleaning is referred to as residual pressure drop, which is an important parameter for the evaluation of fabric filtration performance. The testing result of residual pressure drop is shown in Fig.3. At the end of the test, the test filter is removed and weighed to obtain the residual dust load (Fig.4).

In Fig.3, the VDI type-1 rig and JIS rig give nearly the same residual pressure drop in the first 30 cycles of Step 1 before the filter aging condition. However, after the aging condition, the residual pressure drop in the last 30 cycles of Step 4 with JIS rig is much higher than that with VDI type-1 rig, indicating that the filter cleaning efficiency with JIS rig is considerably lower than that with VDI type-1 rig. The difference in the cleaning efficiency between the rigs is also clearly seen from the residual dust load at the end of performance test shown in Fig.4.

The development of residual pressure drop is influenced by not only the filter cleaning efficiency but also the number of pulse jet cleaning. For a new filter before the aging, the particles collected on the filter are relatively easy to be cleaned so that the difference in filter cleaning 
efficiency between VDI type-1 rig and JIS rig would not cause any difference in the residual pressure drop. However, once the filter is aged by applying 5000 cycles of filtration cycles, the particles on the filter become more difficult to be detached and therefore even a small difference in the filter cleaning efficiency leads to a big difference in the residual pressure drop for the last 30 cycles.

Pressure drop during pulse jet cleaning

In order to compare the filter cleaning performance between VDI type-1 rig and JIS rig, the time change in pressure upstream and downstream of the filter during the pulse jet cleaning is measured. Fig.5 shows the pressure evolution during the pulse jet cleaning as well as the pressure drop across the filter. Because of the nature of part-flow employed in VDI type-1 rig, the pressures on both sides in VDI type-1 rig are lower than those in JIS rig. The maximum pressure drop is higher for JIS rig at a shorter time period of pulse jet cleaning.

The filter cleaning is a complicated phenomenon, which is influenced by many factors such as the force acting on a filter, the cleaning air volume, the peak pressure, and the pattern of cleaning pressure buildup. During the filter cleaning, the dust cake detaches from the filter surface at the instant that the force reaches the maximum and a fraction of removed dust will re-attach on the filter surface at the end of filter cleaning because of the reversed gas flow toward the filter surface. The filter cleaning efficiency is therefore affected by the combination of these detachment and re-attachment mechanisms. The detachment of particles is enhanced with the peak pressure and the re-attachment is reduced with increasing the time period of pulse jet.

The differences in configuration of the pulse jet system employed in VDI type-1 rig and JIS rig result in the different filter cleaning performance. In VDI type-1 rig, the pulse jets are ejected through a nozzle with $3 \mathrm{~mm}$ in diameter toward the downstream side of the test filter at a distance of $625 \mathrm{~mm}$ downstream of the filter. The rig volume of the clean side is $13 \mathrm{~L}$. However, in the JIS rig, the nozzle with $9 \mathrm{~mm}$ in diameter faces downward in the horizontal duct. The filter cleaning is performed by the airflow reflected on the duct wall. The distance between the nozzle and the filter is $150 \mathrm{~mm}$ and the rig volume of the clean side is $29 \mathrm{~L}$. The differences in the configuration of two rigs make it more difficult to compare the filter cleaning performance.

\section{APPLICATION OF NEW MODEL FOR FILTER CLEANING PERFORMANCE EVALUATION} A model for fabric filter

Kanaoka and Yao ${ }^{[6]}$ recently studied the filtration phenomenon with a pulse jet non-woven fabric filter. They found that the evolution of pressure drop of a flat type fabric filter could be divided into three stages in a filtration cycle, where the increasing rate of pressure drop gradually increases in the first stage and then decreases in the second stage, and finally it is almost a constant in the third stage as shown in Fig.6. Based on this study, we propose a new model, dividing the fabric filtration process into three stages, i.e. depth filtration stage, transition filtration stage and surface filtration stage.

After filter cleaning, the residual dust cake remains on a portion of filter surface, whereas on 
a portion of filter surface, not only the dust cake is completely removed but also a part of dust inside the filter is also cleaned. Therefore, the depth filtration takes place on the cleaned surface just after the filter cleaning until the dust load reaches a maximum retention in the filter. Since the filtration velocity through the cleaned surface is much higher than that through the un-cleaned surface, we assume that there is airflow only through the cleaned surface.

Once the dust begins to accumulate on the cleaned surface, which is the end of depth filtration stage, the second stage of transition filtration stage begins. During the transition stage, the surface filtration on the un-cleaned surface also plays a role for dust collection. The local filtration velocity varies over the filter surface because of the non-uniformity in the dust cake distribution. As time passes, the non-uniformity will gradually disappear because of self-equalizing mechanism for parallel path flows. The difference in local filtration velocity will then diminish and this will lead to uniform deposition of filter cake, which is the beginning of surface filtration stage.

Surface cleaning fraction and residual dust load on un-cleaned surface

In order to express the non-uniformity of residual dust cake distribution after filter cleaning in a simpler manner, the present model introduces two parameters, i.e. the surface cleaning fraction, $f$, which is the ratio of cleaned surface area to the total surface area of a filter, and the residual dust load on un-cleaned surface after cleaning, $W_{u 0}$. Here "cleaned" implies that the dust cake accumulated on the filter is completely removed and yet the dust inside the filter is also partially cleaned. The residual dust distributes homogenously on the un-cleaned surface. Both $W_{u 0}$ and $f$ change with the number of filtration cycle.

According to our model the pressure drop development during the transition and surface filtration stages can be expressed as a function of $f, W_{u 0}$ and $t$. In order to obtain the surface cleaning fraction, $f_{i-1}$, and the residual dust load on the un-cleaned surface after cleaning, $W_{u 0, i-1}$, two points on the experimental curve of $\Delta p_{s}$ at $t\left(t=\tau_{i}\right.$ and $\left.t=\tau_{i}+\psi_{i}\right)$ are utilized, where $\tau_{i}$ is the duration of depth filtration period and $\psi_{i}$ is the duration of transition and surface filtration periods for the $i$-th cycle. At $t=\tau_{i}$, Eq.(1) can be obtained since $R_{u \tau, i}>>R_{c \tau, i}$.

$$
\Delta p_{\tau, i}=\mu \frac{u}{f_{i-1}} R_{c \tau, i}
$$

At $t=\tau_{i}+\psi_{i}$, the pressure can be approximately expressed by:

$$
\Delta p_{\max }=\mu u\left(R_{c \tau, i}+K\left(1-f_{i-1}\right) W_{u 0, i-1}+K c_{0} u \psi_{i}\right)
$$

If we assume that Eq.(1) holds for a short time period, $\Delta t$, in the beginning of transition filtration, then we have:

$$
\Delta p_{\tau+\Delta t, i}=\mu \frac{u}{f_{i-1}}\left(R_{c \tau, i}+K c_{0} \frac{u}{f_{i-1}} \Delta t\right)
$$

where the second term in the bracket is the increase in resistance due to the particles accumulated on cleaned surface. Subtracting Eq.(1) from Eq.(3) and letting $\Delta t \rightarrow 0$, we have:

$$
\lim _{\Delta t \rightarrow 0} \frac{\Delta p_{\tau+\Delta t, i}-\Delta p_{\tau, i}}{\Delta t}=\left[\frac{d \Delta p}{d t}\right]_{\tau, i}=\frac{\mu K c_{0} u^{2}}{f_{i-1}^{2}}
$$


Since $\left[\frac{d \Delta p}{d t}\right]_{\tau, i}=\left[\frac{d \Delta p}{d t}\right]_{\max , i}, f_{i-1}$ can be calculated by:

$$
f_{i-1}=\left(\mu K c_{0} u^{2} /\left[\frac{d \Delta p}{d t}\right]_{\max , i}\right)^{1 / 2}
$$

Now we have $f_{\mathrm{i}-1}, W_{u 0, i-1}$ can then be calculated by Eq.(3) :

$$
W_{u 0, i-1}=\frac{\frac{\Delta p_{\max }-f_{i-1} \Delta p_{\tau, i}}{\mu u}-K c_{0} u \psi_{i}}{K\left(1-f_{i-1}\right)}
$$

Evaluation on filter cleaning performance by the model

Utilizing the filter performance test results with the VDI type-1 and JIS rigs, $f$ and $W_{u 0}$ are calculated and plotted in Figs. 7 and 8. Fig.7 shows there is no significant difference in $f$ by the rigs employed. However, in Fig.8, $W_{u 0}$ obtained with JIS rig is considerably higher than that with VDI type-1 rig for the last 30 cycles after the aging, indicating that the dust cleaning efficiency is higher for the VDI type-1 rig. This is in accordance with the results shown in Figs.3 and 4. Therefore, the difference in filter cleaning performance can be expressed in terms of $f$ and $W_{u 0}$ by our model.

Residual pressure drop vs. outer residual dust load

Residual dust load consists of two parts, i.e. inner residual load and outer residual dust load. The outer residual dust load is determined by $f$ and $W_{u 0}$ as:

$$
W_{\text {out }}=W_{u 0} /(1-f)
$$

The outer residual dust load is obtained from the calculation results in Figs. 7 and 8 and it is plotted with residual pressure drop together in Fig.9. It is shown that the residual pressure drop increases with the outer residual dust load in the same way for the two rigs either before aging or after aging. The compatibility of the VDI type-1 rig and JIS rig is verified by the model.

\section{CONCLUSIONS}

1. VDI type-1 rig gives a higher filter cleaning performance than JIS rig.

2. During the pulse jet cleaning, the peak pressure measured with JIS rig is higher than that with VDI type-1 rig, and the time period of pulse jet with JIS rig is shorter than that with the VDI type-1 rig.

3. The difference in filter cleaning performance measured by the VDI type-1 and JIS rigs can be expressed in terms of $f$ and $W_{u 0}$ according to our model.

\section{NOMENCLATURE}

$c_{0}:$ Inlet dust concentration $\left(\mathrm{kg} / \mathrm{m}^{3}\right)$

$f$ : Surface cleaning fraction (-)

$K$ : Specific resistance of accumulated dust on filter $(\mathrm{m} / \mathrm{kg})$ 
$\Delta p$ : Filter pressure drop $(\mathrm{Pa})$

$R:$ Resistance of filter $(1 / \mathrm{m})$

$t:$ Filtration time (s)

$u:$ Filtration velocity $(\mathrm{m} / \mathrm{s})$

$W$ : Dust load per unit surface area of filter $\left(\mathrm{kg} / \mathrm{m}^{2}\right)$

$\mu$ : Gas viscosity $(\mathrm{Pa} \cdot \mathrm{s})$

$\tau$ : Duration of depth filtration period (s)

$\Psi$ : Duration of transition and surface filtration periods (s)

Subscript

c : Cleaned surface

i : Number of filtration cycle

max : Maximum

out : Out

$\mathrm{s}:$ Transition and surface filtration stages

$\mathrm{u}$ : Un-cleaned surface

$\tau:$ At the end of depth filtration

\section{REFERENCES}

1. VDI/DIN 3926 "Testing of Cleanable Filter Media"

2. JIS Z 8909-1 "Testing methods of filter media for dust collection"

3. W. Duo, N.F. Kirkby, J.P.K. Seville, R. Clift, Patchy Cleaning of Rigid Gas Fitlers - I . A Probabilistic Model, Chemical Engineering Science, 52 (1) (1997) 141-151.

4. W. Duo, J.P.K. Seville, N.F. Kirkby, H. Buchele, C.K. Cheung, Patchy Cleaning of Rigid Gas Filters-II. Experiments and Model Validation, Chemical Engineering Science, 52(1) 1997 153-164.

5. A. Dittler, G. Kasper, Simulation of Operational Behavior of Patchily Regenerated, Rigid Gas Cleaning Filter Media, Chemical Engineering and Processing, 38 (1999) 321-327.

6. C. Kanaoka, Y.P. Yao, Time Dependency of the Pressure Drop in a Flat Type Pulse Jet Fabric Filter, Kagaku Kogaku Ronbunshu, 29(2) 2003 267-271. in Japanese 


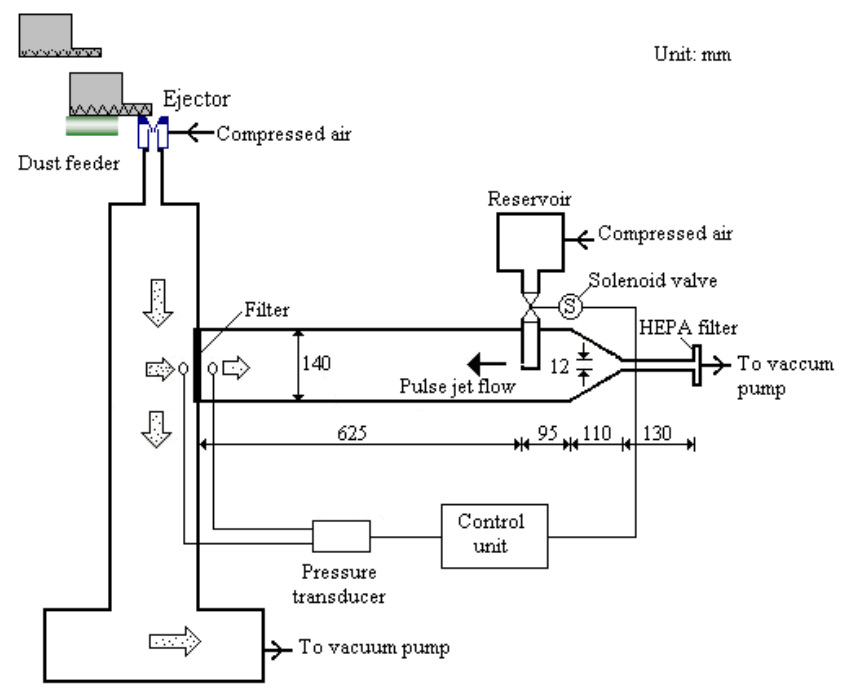

Fig. 1 Schematic diagram of VDI type-1 rig

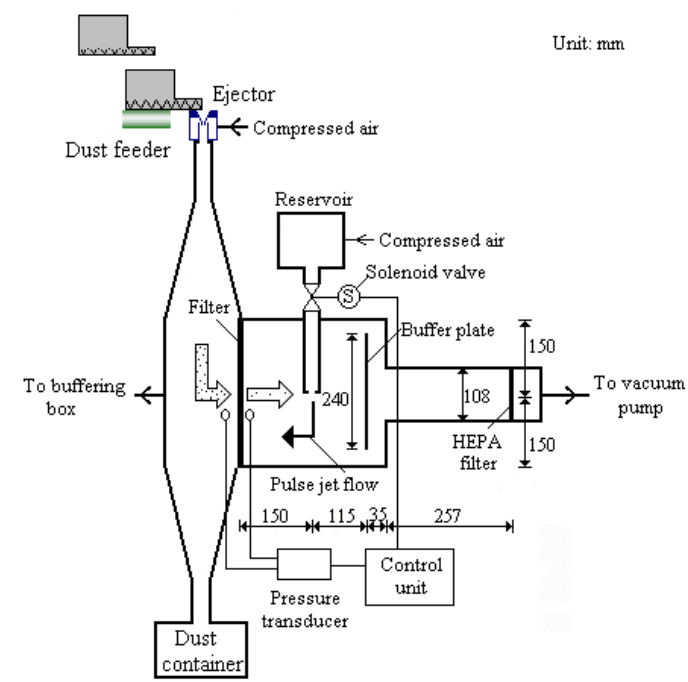

Fig. 2 Schematic diagram of JIS rig

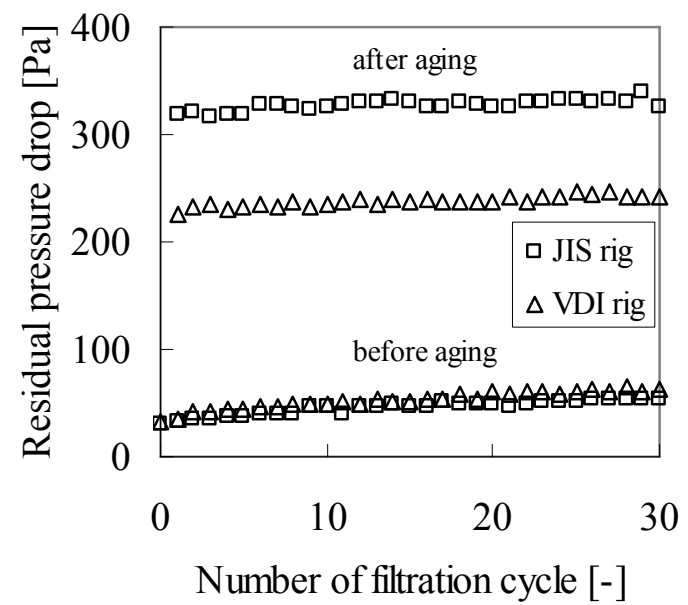

Fig.3 Residual pressure drop 


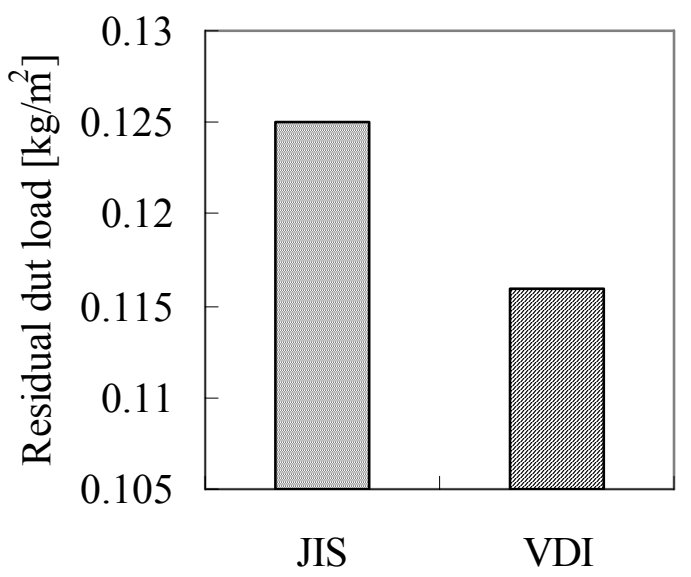

Fig.4 Residual dust load at the end of performance test



Fig.5 Pressure drop development during pulse jet cleaning 


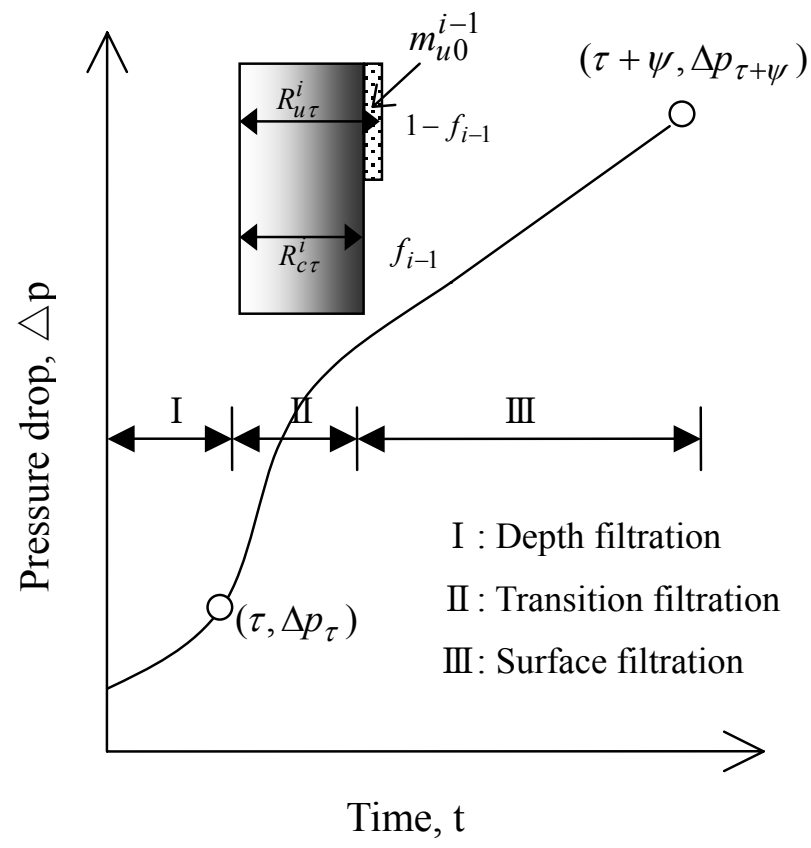

Fig. 6 Illustration on a filtration cycle of fabric filter

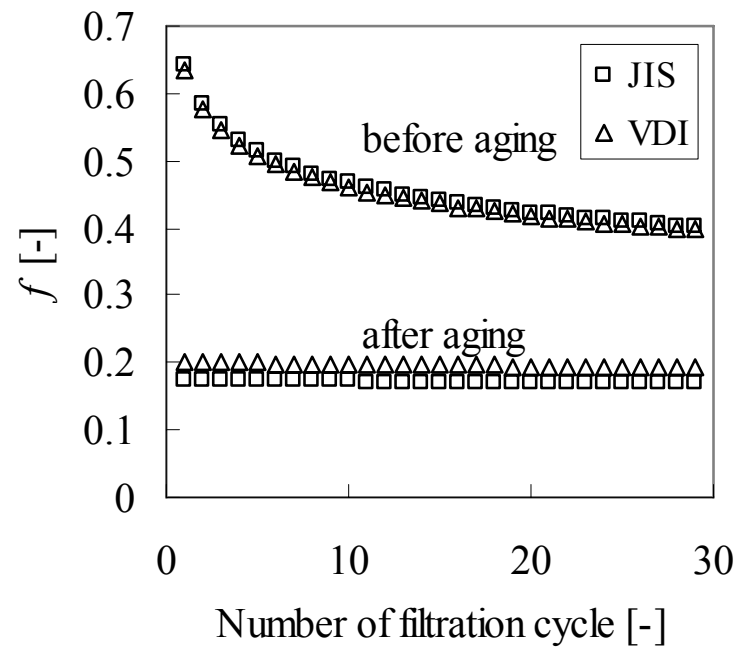

Fig.7 Surface cleaning fraction 


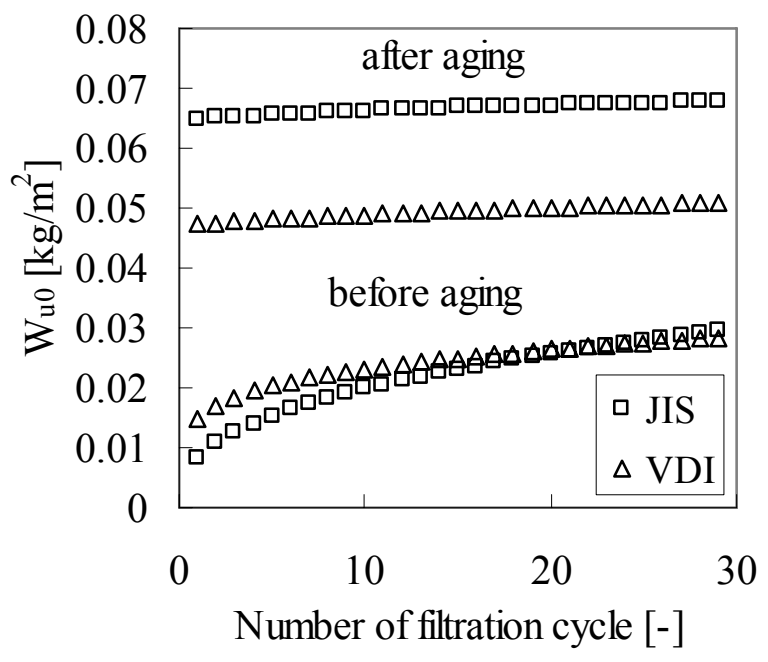

Fig.8 Residual dust load on un-cleaned surface

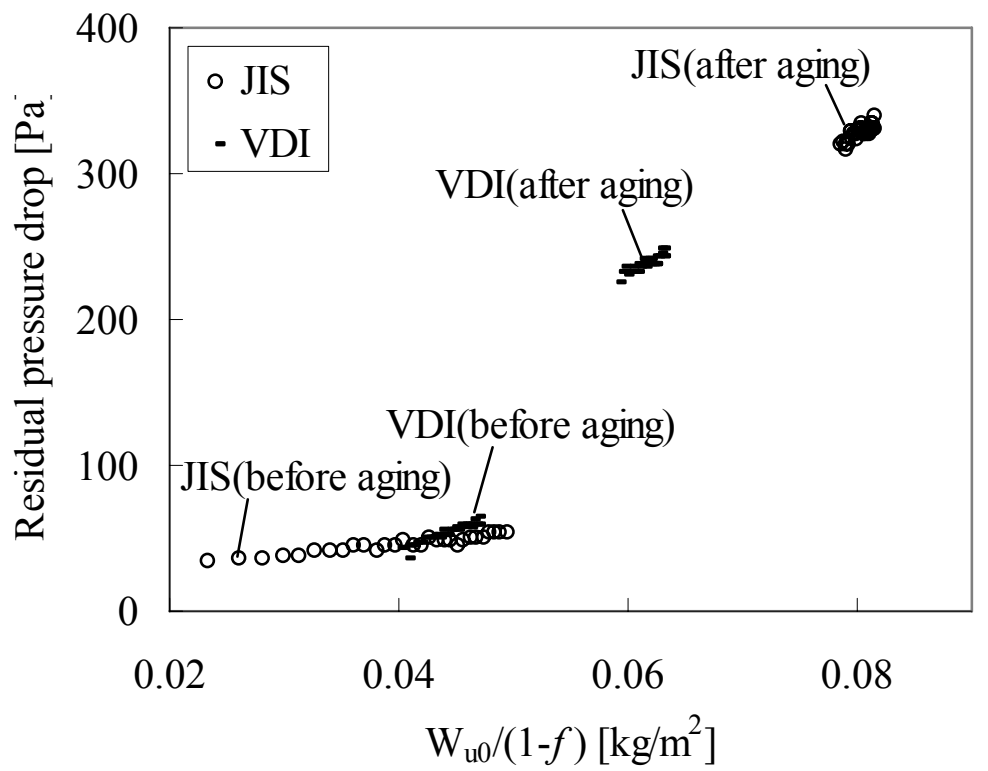

Fig. 9 Residual pressure drop vs. outer residual dust load 
Table 1 Testing conditions

\begin{tabular}{|c|c|c|}
\hline \multirow{2}{*}{ Fabric filter } & Material: & PE singed \\
\hline & Structure: & Felt \\
\hline \multirow{4}{*}{ Dust } & Particle material: & Pural NF \\
\hline & Mass median diameter: ${ }^{*}$ & 5.8 \\
\hline & Density: & $600-800$ \\
\hline & Dust concentration: & 5 \\
\hline \multirow{3}{*}{ Testing conditions } & Filtration velocity: & 0.033 \\
\hline & Pulse jet pressure: & 500 \\
\hline & Filter pressure before cleaning: $\quad[\mathrm{Pa}]$ & 1000 \\
\hline
\end{tabular}

* Mass median diameter was measured with Sympatec HELOS 